\title{
a) XDR
}
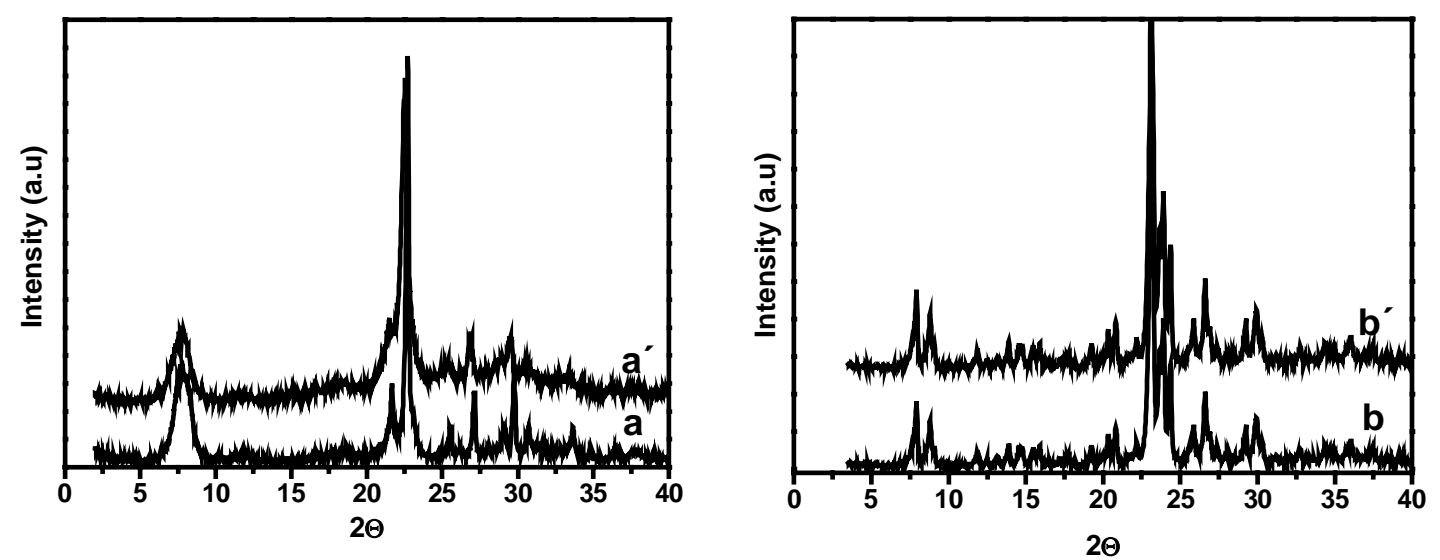

Fig.1. XDR patterns of cobalt Beta (as-synthesized (a) and calcined (a')) and cobalt ZSM - 5 (as-synthesized (b) and calcined ( $\left.b^{\prime}\right)$ ). The XDR spectra only display the peaks of Beta and ZSM-5 zeolites, showing that the method used produces Beta and ZSM - 5 with Co highly dispersed in the inorganic framework.

b) UV-Vis
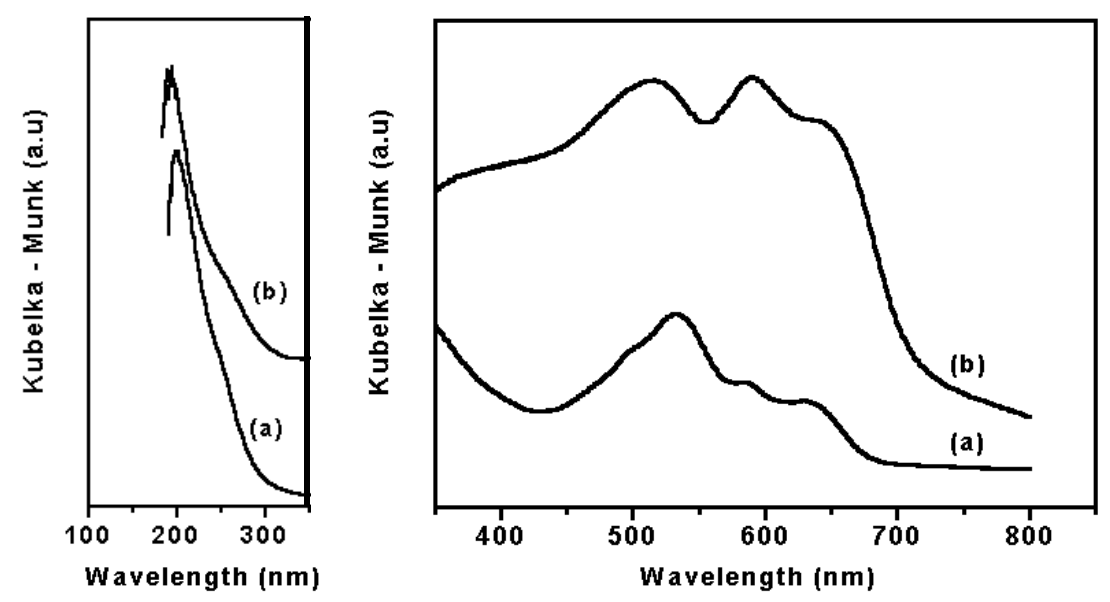

Fig.2. UV-Vis spectra of BetaCo a) as synthesized, and b) calcined. The spectra display a band at $210 \mathrm{~nm}$ and a triplet at 530, 590 and $640 \mathrm{~nm}$ corresponding to tetragonal cobalt. Calcination at $853 \mathrm{~K}$ does not change the general trends of the spectrum, only the relative intensities of the triplet bands are modified. 


\section{c) Low Resolution TEM}

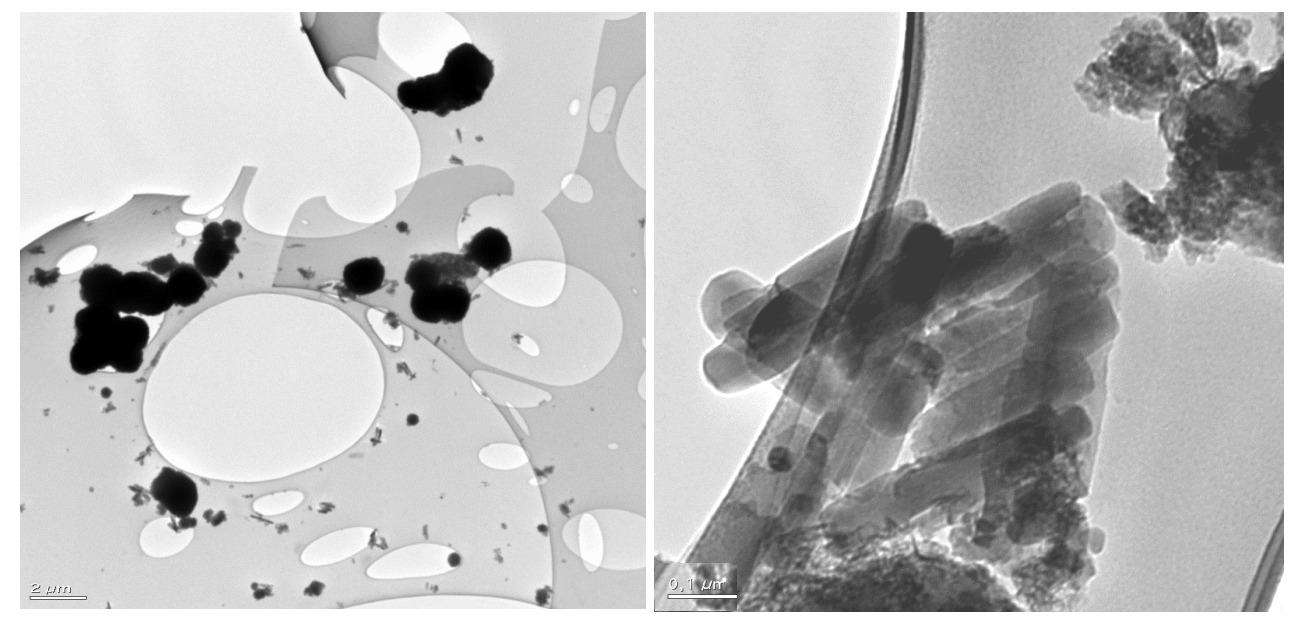

Fig.3. TEM image of the BetaCo sample. Low and moderate resolution images of BetaCo grains dispersed onto a holey carbon gird.

\section{d) Fitting of the magnetization loops.}

The hysteresis loops at temperatures between the mean blocking $T\left(T_{\max }\right)$, and $30 \mathrm{~K}$ allow us to extract the magnetic moment per particle when fitting Langevin functions

$$
M(H, T)=\int_{0}^{\infty} D^{3} L\left(\frac{\mu(D) H}{k_{B} T}\right) P(D) d D,
$$

where $D$ is the cluster diameter, $L(\mathrm{x})$ is a classical Langevin function and $P(D)$ is the size distribution. The fit perfectly correlates with the experimental data at all measured $T$. That fit to Langevin functions at all measured $T$ indicates that the whole magnetization is coming from narrowly dispersed small ferromagnetic particles, and therefore that the matrix magnetic signal is nil. From the total magnetic moment of these samples one can estimate that only one $20^{\text {th }}$ of the present $\mathrm{Co}_{\mathrm{o}}$ is participating in the magnetization, i.e., a $0.25 \mathrm{wt} \%$ of the sample

\section{e) Longer Heat Treatments}

Longer heat treatments leads to a population of smaller and larger particles and a broader peak in the ZFC centred at lower T (Fig.4). The small particles are smaller than before because the matrix where they are coming from has a 
smaller content on Co. The irreverisble Temperature $T_{\text {irr }}$ (the point where the ZFC and the FC curves split) is shifted to higher T, far from the maximum, indicating the presence of larger magnetic particles in the sample.

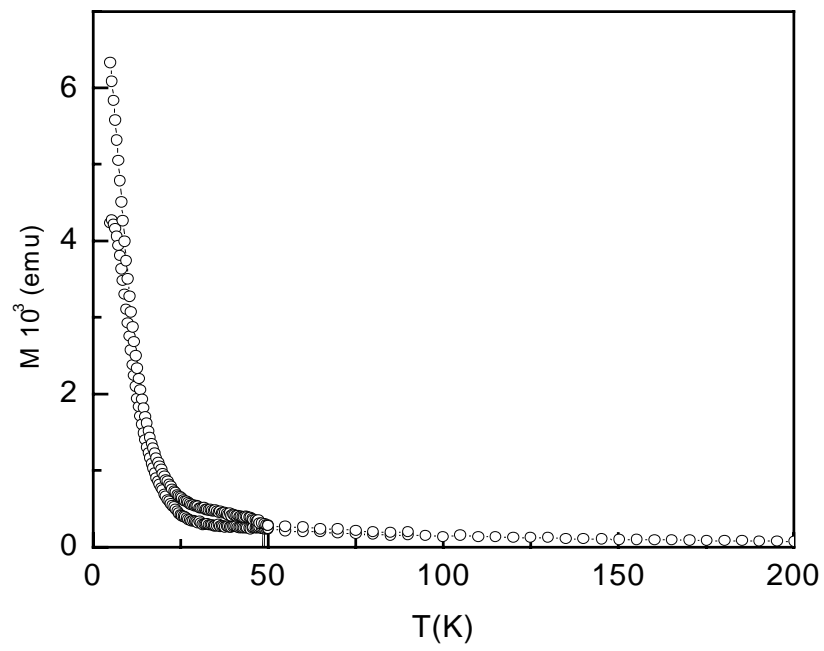

Fig.4. ZFC/FC magnetization for the annealed sample up to $200 \mathrm{~K}$. The sample was heated for 5 hours at $750 \stackrel{\circ}{\circ}$ (including 3\%/min heating ramp) under $\mathrm{N}_{2}$ and 6 hours at $750^{\circ} \mathrm{C}$ open to air.
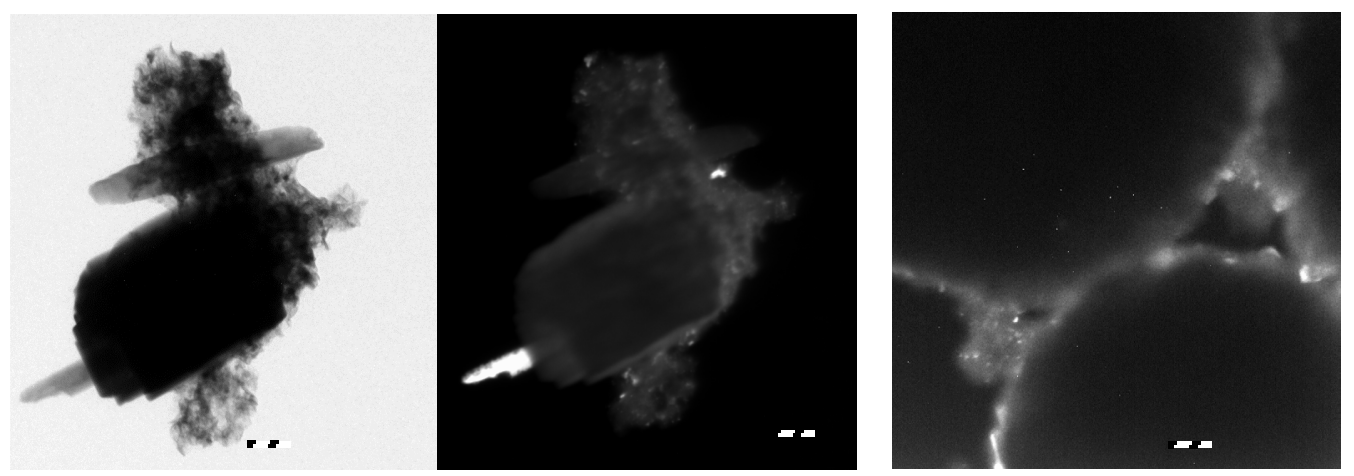

Fig.5. TEM images of a sample exposed to a longer heat treatment. (Left) bright field image of a zeolite containing cobalt grain. (Center) Dark field of the same grain where the cobalt particles are highlighted. The Dark field technique is sensitive to more dense and crystalline areas, in this case corresponding to cobalt and cobalt oxide particles, which appear bright in a dark background. (Right) Dark field of zeolite grains. cobalt crystals can be observed in the shores of the grains. Bars are $100 \mathrm{~nm}$. 
The longer heat treatments also result in the presence of $\mathrm{Co}-\mathrm{CoO}$ mixed crystals displaying exchange coupling and exchange bias. This is clearly observed in an increased coercivity and increase in the irreversibility field (where the hysteresis loop closes) and, mainly, a shift of the hysteresis loop, after field cooling, in the opposite direction of the applied field (10 kOe) during cooling (Fig.6). Same experiment in the calcined samples did not show any of these features. At intermediate longer heat treatments, intermediate results were observed, as shown in Fig. 7.

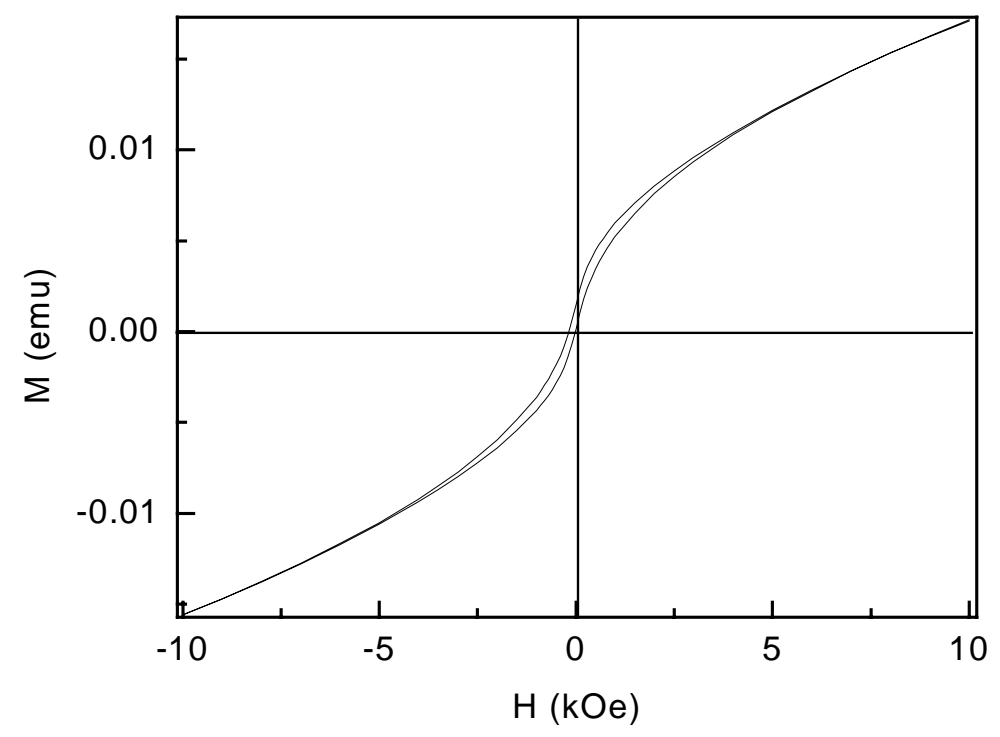

Fig.6. Hysteresis loops at $5 \mathrm{~K}$ after $10 \mathrm{kOe}$ field cooling. 


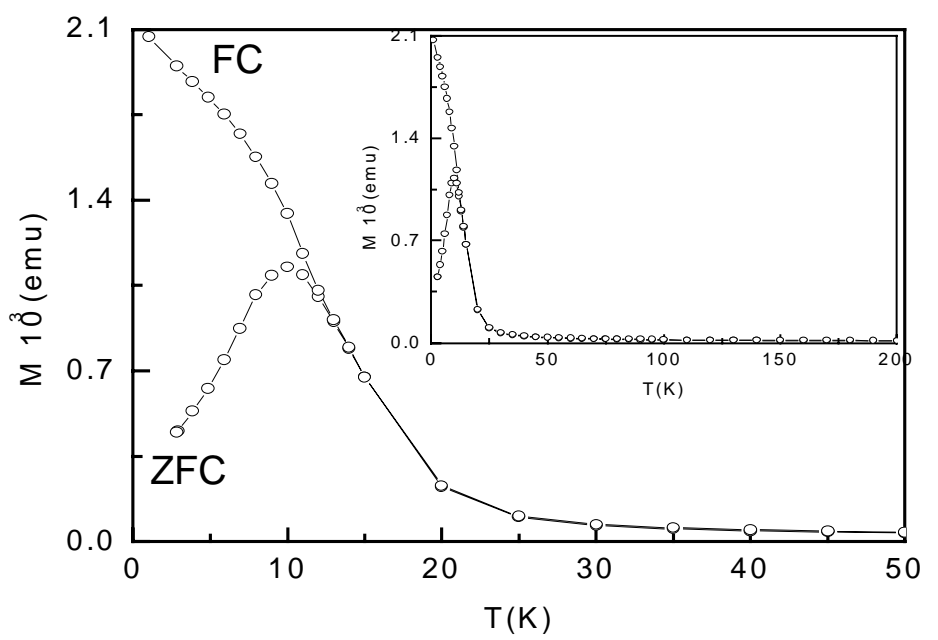

Fig.7. ZFC/FC magnetization for the intermediate annealed sample. The sample was heated for 5 hours at $750^{\circ} \mathrm{C}$ (including 3\%/min heating ramp) under $\mathrm{N}_{2}$ and 6 hours at $750 \stackrel{\circ}{\circ}$ open to air. Compared to the former samples, here the ZFC peak broadens and $T_{\text {irr }}$ is shifted towards values higher than $T_{B}$.

\section{f) BetaCo vs ZSM5Co after calcination}

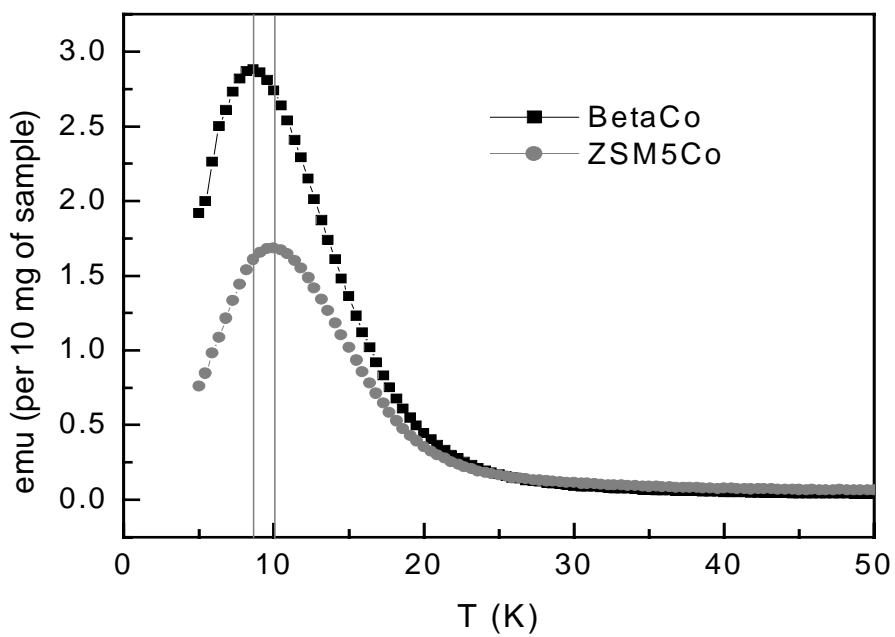

Fig.8. ZFC/FC magnetization for the BetaCo and ZSMCo samples. 


\section{g) Complete list of authors citations with more than 10 authors.}

(1) Alivisatos, A. P.; Barbara, P. F.; Castleman, A. W.; Chang, J.; Dixon, D. A.;

Klein, M. L.; McLendon, G. L.; Miller, J. S.; Ratner, M. A.; Rossky, P J.; Stupp, S. I.; Thompson, M. E.

(3) Respaud, M.; Broto, J. M.; Rakoto, H.; Fert, A. R.; Thomas, L.; Barbara, B.; Verelst, M.; Snoeck, E.; Lecante, P.; Mosset, A.; Osuna, J.; Ely, O.; Amiens, C.; Chaudret, B.

(6) Crespo, P.; Litran, R.; Rojas, T. C.; Multigner, M.; de la Fuente, J. M.; Sánchez-López, J. C.; García, M. A.; Hernando, A.; Penades, S.; Fernández, A.

(23) Gambardella, P.; Rusponi, S.; Veronese, M.; Dhesi, S. S.; Grazioli, C.; Dallmeyer, A.; Cabria, I.; Zeller, R.; Dederichs, P. H.; Kern, K.; Carbone, C.; Brune, $\mathrm{H}$. 